\title{
Erratum to: Irritable bowel syndrome: is it associated with genotypes of Blastocystis hominis
}

\author{
Javed Yakoob • Wasim Jafri • Mohammad Asim Beg • \\ Zaigham Abbas $\cdot$ Shagufta $\mathrm{Naz} \cdot$ Muhammad Islam • \\ Rustam Khan
}

Published online: 8 October 2011

(C) Springer-Verlag 2011

\section{DOI 10.1007/s00436-010-1761-x}

The original version of this article unfortunately contained a mistake. In Table 2 we calculated the percentages presented for the stool microscopy and culture of $B$. hominis row wise rather than vertically in the column. As this was aimed to determine the distribution of B.hominis in IBS-D versus control group, we should have calculated the percentage column wise. However, percentages calculated row wise

The online version of the original article can be found at http://dx.doi. org/10.1007/s00436-010-1761-x.

J. Yakoob $\cdot$ W. Jafri $\cdot$ Z. Abbas $\cdot$ S. Naz $\cdot$ R. Khan

Department of Medicine, The Aga Khan University,

Stadium Road,

Karachi, Pakistan

\section{A. Beg}

Department of Pathology and Microbiology,

The Aga Khan University,

Karachi, Pakistan

\section{Islam}

Department of Medicine and Community Health Sciences,

The Aga Khan University,

Karachi, Pakistan

\section{J. Yakoob $(\bowtie)$}

Department of Medicine, The Aga Khan University Hospital,

Stadium Road,

Karachi 74800, Pakistan

e-mail: yakoobjaved@hotmail.com will not change the $\mathrm{P}$ value when calculated column wise. There was an error on our part that percentages presented for the stool microscopy and culture of $B$. hominis in the Table 2 should have been reported column wise. Please see amended Table 2.

Table 2 Details of patients enrolled in the study

\begin{tabular}{|c|c|c|c|c|}
\hline & & $\begin{array}{l}\text { IBS-D } \\
n=158(50.2)\end{array}$ & $\begin{array}{l}\text { Control } \\
n=157(49.8)\end{array}$ & $P$ value \\
\hline \multirow[t]{2}{*}{ Age } & Mean \pm SD & $41 \pm 15$ & $42 \pm 14$ & \\
\hline & Range & $16-83$ & $15-75$ & \\
\hline \multicolumn{5}{|l|}{ Gender } \\
\hline & Male & $109(69)$ & $104(66)$ & \\
\hline & Female & $49(31)$ & $53(34)$ & \\
\hline \multicolumn{5}{|c|}{ Stool microscopy for B.hominis } \\
\hline \multirow{2}{*}{\multicolumn{2}{|c|}{$\begin{array}{l}\text { Positive } \\
\text { Negative }\end{array}$}} & $87(55)$ & $32(20)$ & $<0.001$ \\
\hline & & $71(45)$ & $125(80)$ & \\
\hline \multicolumn{5}{|c|}{ Culture for B.hominis } \\
\hline \multirow{2}{*}{\multicolumn{2}{|c|}{$\begin{array}{l}\text { Positive } \\
\text { Negative }\end{array}$}} & $95(60)$ & $38(24)$ & $<0.001$ \\
\hline & & $63(40)$ & $119(76)$ & \\
\hline \multicolumn{5}{|l|}{ Genotype } \\
\hline \multicolumn{5}{|l|}{ Positive } \\
\hline Type 1 & $n=87$ & $75(86)$ & $12(14)$ & $<0.001$ \\
\hline Type 2 & $n=10$ & $6(60)$ & $4(40)$ & 0.487 \\
\hline Type 3 & $n=49$ & $23(47)$ & $26(53)$ & $<0.001$ \\
\hline Type 4 & $n=8$ & $6(75)$ & $2(25)$ & 1 \\
\hline Type 5 & $n=7$ & $3(43)$ & $4(57)$ & 0.197 \\
\hline Type 6 & $n=6$ & $3(50)$ & $3(50)$ & 0.365 \\
\hline Type 7 & $n=10$ & $5(50)$ & $5(50)$ & 0.282 \\
\hline Negative & 181 & $64(35)$ & $117(65)$ & \\
\hline Untypeable & 2 & $2(100)$ & 0 & \\
\hline
\end{tabular}

